\title{
Elemente des Cometen 1884 Barnard.
}

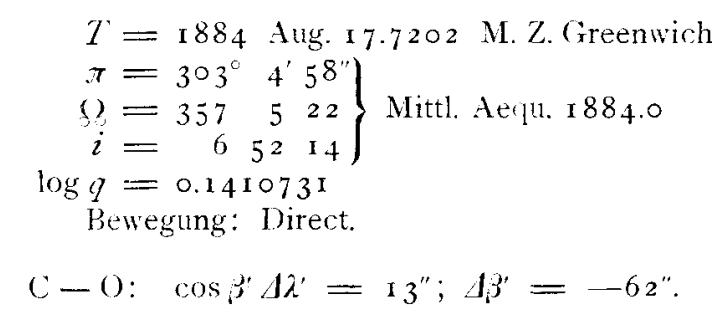

Zur Berechnung der obigen Elemente dieses Cometen benutzte ich die folgenden Beobachtungen:

$\begin{array}{ccc}\text { Nashville, 'Tenn. } & \text { Juli } & \text { 1 } 6 \\ & \Rightarrow & \mathbf{2 2} \\ \text { Cambridge, Mass. } & \gg & 28\end{array}$

Die oben angegebenen Differenzen können durch weitere
Variation von $M$ nicht vermindert werden; die Bahn weicht daher von der einer Parabel bedeutend ab. Durch die geringe Bewegung in Declination wurde die Berechnung der Bahn nicht wenig erschwert.

Ich war deshalb genöthigt, selbst einige Formeln aufzustellen, welche für die Berechnung von Bahnen, in welchen die Bewegung so klein ist wie hier, denjenigen von Olbers und Gauss vorzuziehen sind. Ich werde dieselben demmächst zur Veröffentlichung bringen. Ausserdem gedenke ich die Elemente der elliptischen Bahn sofort zu berechnen.

Ich kann diese Mittheilung nicht schliessen, ohne Herrn W. C. Winlock rom hesigen Marine-Observatorium meinen Dank für die Bereitwilligkeit auszusprechen, mit welcher er mich mit der einschlägigen I.iteratur unterstuitzte. Ohne seine freundliche Hülfe wäre es mir kaum möglich gewesen, in den Besitz des nöthigen Materials zu gelangen.

Columbia University, Washington D. C., I 884 Sept. 3.

Gustave Ravené.

\section{Elliptische Elemente des Cometen 1884 Barnard.}

(Aus einem Schreiben an den Herausgeber.)

Gestatten Sie mir, Ihnen das Resultat meiner Rechnung über den Cometen I 884 Barnard mitzutheilen. Es findet sich dadurch die Vermuthung, dass die Bahn desselben merklich von der Parabel abweiche, vollkommen bestätigt. Zur Berechnung hatte ich benutzt die Beobachtungen zu Algier Juli $23,25,26,27,29$ und daraus unter Vergleichung mit der Ephemeride von Dr. Stechert den Normalort I gebildet. In gleicher Weise ergab sich Normalort II aus den Beobachtungen Aug. 15, 16, 17 zu Nizza, Arcetri und Algier. Mit diesen Orten und einer hiesigen Beobachtung von 1)r. Schur, Sept. 12, berechnete ich erst folgende parabolische Elemente:

$$
\left.\begin{array}{rl}
T & =1884 \text { Aug. } 17.6 \text { 1966 M. Z. Berlin } \\
(1) & =304^{\circ} 17^{\prime} \text { I } 6.3 \\
\Omega & =35^{\prime \prime} \quad 28 \text { 16.5 } \\
i & =653 \quad 0.6
\end{array}\right\} \text { Mittl. Aequ. } 1884.0
$$

Mittlerer Ort: $\Delta \lambda^{\prime \prime}=-\mathbf{x}^{\prime} 4^{\prime \prime \prime} 3 \quad \Delta \beta^{\prime \prime}=+2^{\prime}$ 1 $8^{\prime \prime}$. .

\begin{tabular}{|c|c|c|c|}
\hline & 1884 & $\lambda$ & $\beta$ \\
\hline I & Juli 26.5 & $247^{\circ} 3^{6^{\prime}} 17^{\prime \prime} 9$ & - I $5^{\circ} 54^{\prime} 47^{\prime \prime} 3$ \\
\hline II & Aug. 16.5 & 26 I II I 4.8 & -I 3 I 321.7 \\
\hline III & sept. I 4.5 & $\begin{array}{lll}286 & 3^{6} & 48.9\end{array}$ & 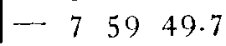 \\
\hline
\end{tabular}

Die Jarstellung des mittl. Ortes deutete auf Ellipticität der Bahn, und so berechnete ich nun, unter Hinzuziehung weiterer Beobachtungen von Sept. I 4 direct eine Ellipse. Jie drei benutzten Positionen sind:

Strassburg I 884 Sept. 18.
Daraus ergahen sich folgende elliptische Elemente:

$$
\begin{aligned}
& T=1884 \text { Aug. } 16.52067 \text { M. 7. Berlin } \\
& \text { (1) }=301^{\circ} 3^{\prime} 40^{\prime \prime} 9 \\
& \left.\Omega=\begin{array}{lll}
9 & 3 & 50.2
\end{array}\right\} \text { Mittl. Aerpu. } 884^{\circ} \circ \\
& i=52849.6 \\
& \varphi=\begin{array}{lll}
3^{6} & 3 & 43.8
\end{array} \\
& a=0.493392 \\
& u=645^{\prime \prime} 533^{6}
\end{aligned}
$$

Umlaufszeit $=2007.4$ Tage

Mittlerer Ort: $\quad 1 \lambda^{\prime \prime} \cos \beta^{\prime \prime}=-\mathrm{I}^{\prime \prime 2} \quad \lambda^{\prime \prime}=-\mathrm{O}^{\prime \prime} \mathrm{I}$.

Die heliocentrischen Coordinaten finden sich aus:

$$
\begin{aligned}
& x=r(9.9999846) \cdot \sin \left(36^{\circ} 6^{\prime} 8^{\prime \prime} 2+v !\right. \\
& y=r(9.9421867) \cdot \sin [306228.6+v] \\
& z=r(9.6844373) \cdot \sin [305 \text { I } 340.3+y]
\end{aligned}
$$

Wie schon Prof. Weiss bemerkte, haben die Elemente des Cometen einige Aehnlichkeit mit denen des Cometen I 844 I de Vico. Nun stimmt auch noch die gefundene Umlaufszeit nahezu mit der von Britnnow für den genannten Cometen berechneten überein. Fine Identität ist aber wohl nicht anzunehmen, da der Zeitraum zwischen I 844 und jetzt keiner ganzen Zahl ron Umläufen entspricht. Auch scheint das Aussehen dieses Coneten dagegen zu sprechen, indem der Comet de Vico in ähnlicher Stellung gegen die Ercle gerade dem blossen Auge sichthar war. Jedenfalls ist der Comet I 884 Barnard zu den interessanten Cometen von kurzer Umlaufszeit zu zählen. 